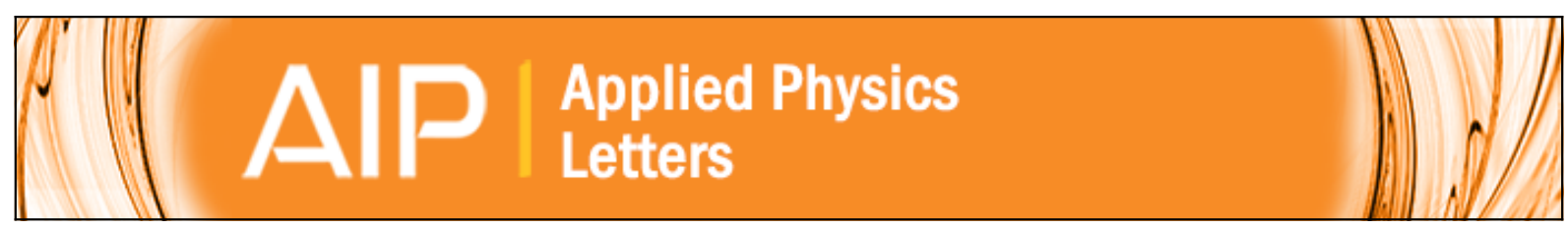

Dielectric waveguide vertically coupled to all-silicon photodiodes operating at telecommunication wavelengths

Kapil Debnath, Frederic Y. Gardes, Andrew P. Knights, Graham T. Reed, Thomas F. Krauss, and Liam O'Faolain

Citation: Applied Physics Letters 102, 171106 (2013); doi: 10.1063/1.4803541

View online: http://dx.doi.org/10.1063/1.4803541

View Table of Contents: http://scitation.aip.org/content/aip/journal/apl/102/17?ver=pdfcov

Published by the AIP Publishing

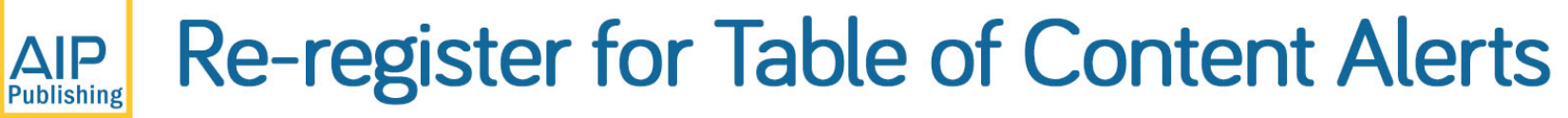




\title{
Dielectric waveguide vertically coupled to all-silicon photodiodes operating at telecommunication wavelengths
}

\author{
Kapil Debnath, ${ }^{1, \text { a) }}$ Frederic Y. Gardes, ${ }^{2}$ Andrew P. Knights, ${ }^{3}$ Graham T. Reed, ${ }^{2}$ \\ Thomas F. Krauss, ${ }^{4}$ and Liam O'Faolain ${ }^{1}$ \\ ${ }^{1}$ SUPA, School of Physics \& Astronomy, University of St Andrews, North Haugh, St Andrews KY16 9SS, \\ United Kingdom \\ ${ }^{2}$ Optoelectronics Research Centre, University of Southampton, Southampton SO17 1BJ, United Kingdom \\ ${ }^{3}$ Department of Engineering Physics, McMaster University, Hamilton, Ontario L8S 4L7, Canada \\ ${ }^{4}$ Department of Physics, University of York, York YO10 5DD, United Kingdom
}

(Received 29 January 2013; accepted 17 April 2013; published online 30 April 2013)

\begin{abstract}
We present a method for detecting light in the $1550 \mathrm{~nm}$ wavelength window based on a silicon nitride waveguide that is vertically coupled to a silicon photonic crystal cavity. The absorption in silicon arises from deep-levels created by ion implantation, thereby providing excellent CMOS compatibility. We demonstrate a responsivity of $0.108 \mathrm{~A} / \mathrm{W}$ at $-10 \mathrm{~V}$ reverse bias with a dark current of $9.4 \mathrm{nA}$. Our work demonstrates one of the smallest wavelength selective photodectors realised to date. By cascading such detectors we also demonstrate a two-channel demultiplexer.
\end{abstract} (C) 2013 AIP Publishing LLC [http://dx.doi.org/10.1063/1.4803541]

Today's electronics industry is confronted with a serious bottleneck due to the prohibitive energy consumption and limited transmission bandwidth of copper interconnects. Energy efficient wavelength division multiplexing (WDM) offers a solution to this problem, especially with siliconbased components that can be integrated monolithically with CMOS circuits and be mass-produced at low cost. ${ }^{1}$

Significant progress in the development of all-silicon based modulators and passive optical components has already been made, yet photodetection typically requires the hybrid integration of other materials such as III-V semiconductors $^{2}$ or germanium, ${ }^{3}$ adding considerable process complexity and cost. An all-silicon approach would clearly be preferable. Due to its large bandgap, however, silicon does not absorb light in the important communications bands around $1310 \mathrm{~nm}$ and $1550 \mathrm{~nm}$. By introducing deep-levels within the bandgap through a standard ion implantation process, it is possible to create absorbing states that can be used for photodetection. ${ }^{4}$ Using this technique, Geis et al. realized a photo-diode with a responsivity of $0.8 \mathrm{~A} / \mathrm{W}$ at $-20 \mathrm{~V}$ based a silicon rib waveguide, highlighting the viability of the approach. ${ }^{5}$ The main drawback is that the absorption coefficient is relatively weak; hence long waveguides with a large footprint are required.

In order to reduce the footprint, resonant enhancement has been applied successfully to reduce the size of deep-level detectors. ${ }^{6}$ Furthermore, resonant devices naturally provide the wavelength selectivity required for demultiplexing WDM channels, thereby simplifying the system and reducing loss. Here, we take this approach further and use a Photonic Crystal (PhC) cavity to reduce the detector length to less than $20 \mu \mathrm{m}$ while achieving high responsivity, $0.108 \mathrm{~A} / \mathrm{W}$ at $-10 \mathrm{~V}$ reverse bias, with $9.4 \mathrm{nA}$ dark current. The cavities are efficiently coupled to a silicon nitride $\left(\mathrm{Si}_{3} \mathrm{~N}_{4}\right)$ waveguide, which forms an integral part of the system; therefore, one can think of the device as a dielectric waveguide with detection

${ }^{a)}$ Electronic mail: kd343@st-andrews.ac.uk capability. PhC cavities are unique in facilitating such efficient coupling between two very different material systems. ${ }^{7}$

A schematic of the experimental realisation of our design is shown in Fig. 1(a). It consists of a PhC cavity embedded into a pin diode. $\mathrm{A} \mathrm{Si}_{3} \mathrm{~N}_{4}$ based bus waveguide is placed vertically above the cavity and separated by a buffer layer. This buffer layer acts as a physical separation between the waveguide mode and the cavity mode. Light is launched at one end of the waveguide and couples to the cavity mode when on resonance. Some of the coupled light is lost due to scattering/radiation loss or is absorbed in the cavity, while the remainder couples back to the waveguide mode contributing to the reflection and transmission components in the waveguide. The coupling efficiency between the waveguide mode and the cavity mode can be optimized by maximizing the overlap between the k-space distributions of the two modes, as discussed in Ref. 7. In the weak coupling approximation, the transmittance $(\mathrm{T})$, reflectance $(\mathrm{R})$, and optical loss (L) on resonance can be expressed as ${ }^{8}$
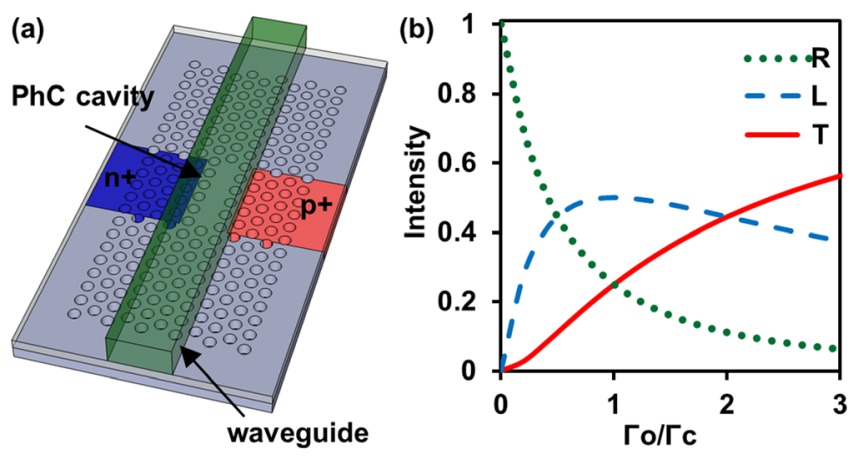

FIG. 1. (a) Schematic of the device, where the defect implanted PhC cavity and the bus waveguide are vertically coupled through a buffer layer. An arbitrary $\mathrm{PhC}$ cavity is shown in the schematic for illustration purposes. The design of Ref. 9 is used in our experiment. (b) Theoretical plot of transmittance $(\mathrm{T})$, reflectance $(\mathrm{R})$, and optical loss $(\mathrm{L})$ against $\Gamma_{\mathrm{O}} / \Gamma_{\mathrm{C}}$ at resonance wavelength, using coupled mode theory. 


$$
\mathrm{T}=\frac{\left(\Gamma_{O}\right)^{2}}{\left(\Gamma_{O}+\Gamma_{C}\right)^{2}} ; \quad \mathrm{R}=\frac{\left(\Gamma_{C}\right)^{2}}{\left(\Gamma_{O}+\Gamma_{C}\right)^{2}} ; \quad \mathrm{L}=1-\mathrm{T}-\mathrm{R},
$$

where $\Gamma_{\mathrm{C}}$ represents the decay rate of the cavity mode into the waveguide mode, which corresponds to the coupling strength between the two modes and $\Gamma_{O}$ represents the decay rate of the uncoupled cavity mode due to scattering/ radiation $\left(\Gamma_{\mathrm{S}}\right)$ and/or absorption $\left(\Gamma_{\mathrm{A}}\right)$ in the cavity, where $\Gamma_{\mathrm{O}}=\Gamma_{\mathrm{S}}+\Gamma_{\mathrm{A}}$. This holds for the standing wave case, ${ }^{8}$ which is appropriate for $\mathrm{PhC}$ cavities. As can be seen in Fig. 1(b), T increases and $R$ decreases with increasing $\Gamma_{\mathrm{O}} /$ $\Gamma_{\mathrm{C}}$. The optimum condition for photon detection occurs when $\Gamma_{\mathrm{C}}=\Gamma_{\mathrm{O}}$ and $\Gamma_{\mathrm{A}} \gg \Gamma_{\mathrm{S}}$. This corresponds to $\mathrm{T}$ and $\mathrm{R}$ values of $0.25(-6 \mathrm{~dB})$ with almost half $(-3 \mathrm{~dB})$ of the incident light being absorbed. This is the critical coupling condition but with a different form to that familiar from ring resonators (see Ref. 8 for a full discussion). Importantly, as long as the scattering loss is much lower than the absorption loss, an internal quantum efficiency of 0.50 may be achieved even with a weak absorber.

The $\mathrm{PhC}$ cavity design used here is a high quality factor cavity and is created by locally modifying the width of a W1 $\mathrm{PhC}$ waveguide. ${ }^{9}$ As a result, both $\Gamma_{\mathrm{S}}$ and $\Gamma_{\mathrm{A}}$ are very small when defect-free silicon is used as the PhC cavity. By introducing lattice defects in the cavity region, it is possible to increase the value of $\Gamma_{\mathrm{A}}$ without affecting $\Gamma_{\mathrm{C}}$ and $\Gamma_{\mathrm{S}}$, and at this point the optical loss will be dominated by defectmediated absorption. Furthermore, by optimizing the concentration of defects, it is possible to achieve $\Gamma_{\mathrm{C}}=\Gamma_{\mathrm{O}}$, which is the optimum situation for achieving maximum absorption and converting the maximum amount of incident light into photocurrent.

The fabrication process was identical to that of Ref. 10, except for the additional defect implantation step. Following contact metallization, the devices were implanted with silicon at $6 \mathrm{MeV}$ and a dose of $3 \times 10^{11} \mathrm{~cm}^{-2}$ to introduce the lattice defects. The devices were tested optically by injecting light from a C-band Agilent tunable laser source into the nitride waveguides via butt coupling. The TE polarisation is selected using a polarisation beam splitting cube. Light was then collected from the other end of the waveguide via a collimating lens coupler and passed via optical fiber to a detector. The IV measurements were performed using a Keithley 6487 Pico ammeter/Voltage source connected to the chip via ground signal ground probe. This allowed for the photocurrent generated to be measured for each wavelength while changing the reverse voltage. The wavelength/photocurrent scans were automated using LABVIEW.

To observe the relationship between transmittance and photocurrent at the resonance wavelength, five different detector designs were realised by varying the $\mathrm{k}$-space overlap between the waveguide and the cavity modes, thus varying $\Gamma_{\mathrm{C}}$ in Eq. (1). We characterise the defect absorption by monitoring the transmittance (Eq. (1)); the transmittance was well below $-6 \mathrm{~dB}$ for the unimplanted devices, as $\Gamma_{\mathrm{C}}$ dominates over $\Gamma_{\mathrm{O}}$. After implantation, the absorption increases and so do the cavity decay rate $\Gamma_{\mathrm{O}}=\Gamma_{\mathrm{S}}+\Gamma_{\mathrm{A}}$ and the transmittance, until $\Gamma_{\mathrm{O}}$ and $\Gamma_{\mathrm{C}}$ balance. Figure 2(a) plots the corresponding
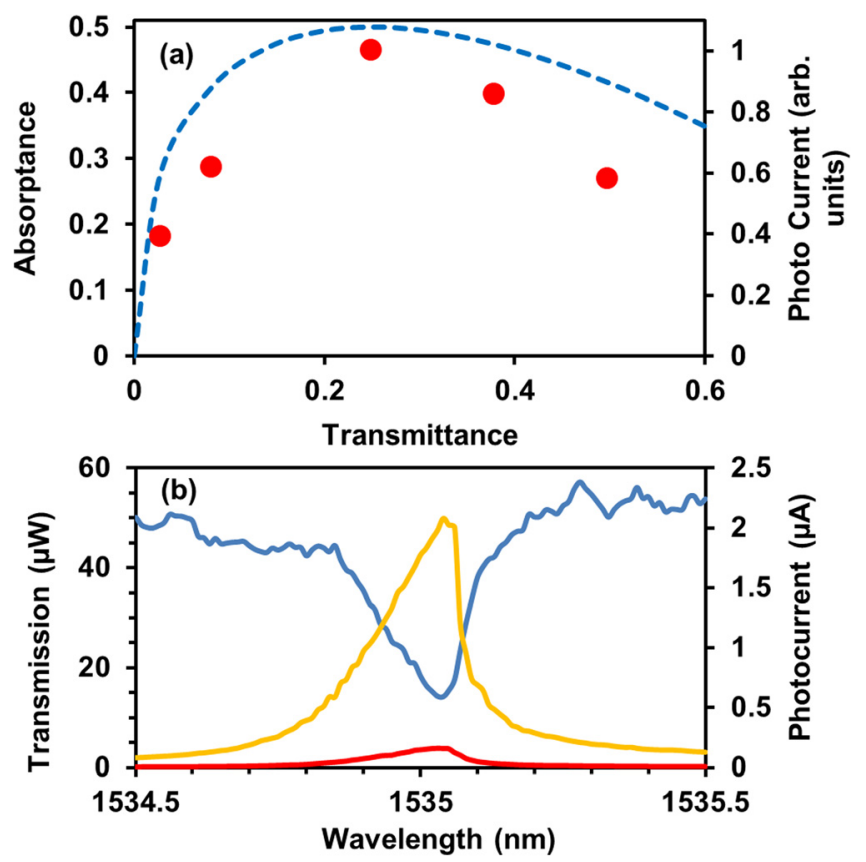

FIG. 2. (a) Blue dashed curve shows the theoretical relationship between transmittance and optical loss of the system at resonance. Red dots show the measured photocurrents for five different detectors with different transmittances. (b) Optical spectra in the $\mathrm{Si}_{3} \mathrm{~N}_{4}$ waveguide immediately after the $\mathrm{PhC}$ cavity (blue) and photocurrent at $0 \mathrm{~V}$ (in red) and $-10 \mathrm{~V}$ (in yellow) for the device with transmittance of 0.30 . The off-resonance optical power is taken from the transmitted power spectrum well away from the resonance.

functional relationship as well as the generated photocurrent for the five different devices with different $\Gamma_{C}$ values. At the implantation dose used here, the propagation loss due to defect absorption is approximately $20 \mathrm{~dB} / \mathrm{cm} .{ }^{11}$ Before defect implantation, the Q-factors of the cavities were around 35000 , roughly corresponding to an equivalent propagation loss of $17 \mathrm{~dB} / \mathrm{cm}$. This loss arises mainly from scattering and free carrier absorption in the heavily doped regions of the pin diode. Thus, after silicon implantation, $\Gamma_{O}$ is dominated by absorption loss as desired. It is clear from Eq. (1) and Fig. 2(a) that the maximum absorption occurs when $\Gamma_{\mathrm{C}}=\Gamma_{\mathrm{O}}$, in which case the transmittance equals $-6 \mathrm{~dB}(0.25)$. In this optimum situation, the maximum amount of light is absorbed and converted into photocurrent.

Figure 2(b) shows the spectrum of the optical power in the waveguide immediately after the $\mathrm{PhC}$ cavity (this is calculated from the measured transmission spectrum using the known losses) and the generated photocurrent for a nearoptimal device. On resonance, the transmittance is 0.30 and by comparing this with the theoretical values in Fig. 1(b), we can estimate the total optical loss in the cavity to be 0.49 $(-3.1 \mathrm{~dB})$. We then define responsivity as the ratio between the generated photocurrent and the total optical loss, which is $-3.1 \mathrm{~dB}$ of the off-resonance optical power $(45 \mu \mathrm{W})$. For $0 \mathrm{~V}$ reverse bias, the generated photocurrent is $0.16 \mu \mathrm{A}$ which corresponds to a detector responsivity of $7 \mathrm{~mA} / \mathrm{W}$ at resonance with a dark current of $0.5 \mathrm{nA}$. For a reverse bias of $-10 \mathrm{~V}$, the photocurrent increases to $2.1 \mu \mathrm{A}$ giving a responsivity of $95 \mathrm{~mA} / \mathrm{W}$, and the dark current becomes $9.4 \mathrm{nA}$. We note that the extinction ratio between the on-resonance photocurrent and off-resonance photocurrent is maintained at $13.8 \mathrm{~dB}$ irrespective of reverse bias. 
Figure 3(a) shows the measured photocurrent versus wavelength, near the resonance wavelength as a function of the off-resonance optical power in the waveguide. The reverse bias is set at $0 \mathrm{~V}$. As the optical power increases, we observe a redshift in the wavelength corresponding to the maximum photocurrent and also an asymmetry in the photocurrent response. We attribute this redshift to the thermo-optic effect caused by carrier recombination inside the intrinsic region of the diode. When the off-resonance optical power increases from $20 \mu \mathrm{W}$ to $85 \mu \mathrm{W}$, the shift in resonance wavelength is $0.18 \mathrm{~nm}$, which corresponds to a change in the cavity temperature of $\sim 2 \mathrm{~K}$ given the thermo-optic coefficient of silicon of $1.86 \times 10^{-4} / \mathrm{K}^{12}$ In addition to thermo-optic detuning, we also note the effect of two-photon absorption, as is apparent in Fig. 3(b), where we plot the peak photocurrent against absorbed optical power. Here, two regions can be identified. For low power operation, the photocurrent increases linearly with absorbed optical power and follows a line corresponding to the $7 \mathrm{~mA} / \mathrm{W}$ responsivity. As the absorbed power increases beyond $20 \mu \mathrm{W}$, a second component with a quadratic dependence becomes apparent, which can be attributed to twophoton absorption. ${ }^{13,14}$ We believe that this behaviour is closely related to that observed in Ref. 15.

We also investigated the effect of reverse bias on photocurrent and resonance wavelength. The off-resonance optical power in the waveguide in this case was $55 \mu \mathrm{W}$, with total optical loss of $25.3 \mu \mathrm{W}$. Figure 3(c) shows the measured photocurrent as the reverse bias is increased from $0 \mathrm{~V}$ to $-10 \mathrm{~V}$. For $0 \mathrm{~V}$ to $-2 \mathrm{~V}$, the photocurrent shows a sharp increase due to the fast sweep-out of photo-generated carriers from the intrinsic region of the diode and then a further (less dramatic) increase with a reverse bias increase from $-2 \mathrm{~V}$ to $-10 \mathrm{~V}$. As shown in Fig. 3(d), the responsivity increases with reverse bias from $9.5 \mathrm{~mA} / \mathrm{W}$ to $108 \mathrm{~mA} / \mathrm{W}$, with a corresponding increase in dark current from $0.5 \mathrm{nA}$ to $9 \mathrm{nA}$.
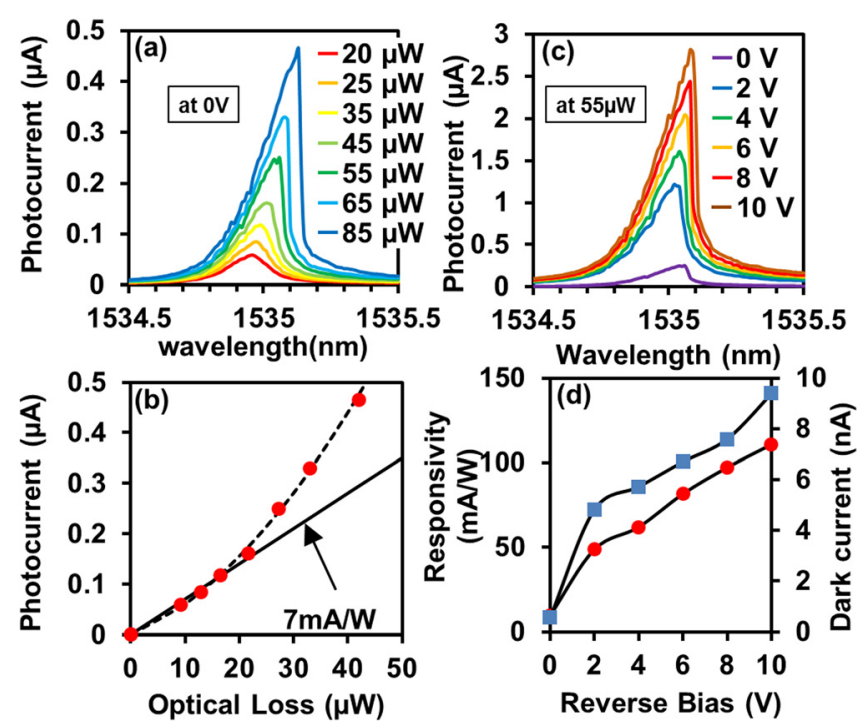

FIG. 3. (a) Measured photocurrent spectra for different input optical power at $0 \mathrm{~V}$ reverse bias. (b) Measured photocurrent peaks as the absorbed optical power increases. The solid line corresponds to $7 \mathrm{~mA} / \mathrm{W}$ responsivity and the dashed line shows the trend in photocurrent as the input power increases. (c) Measured photocurrent spectra for different applied reverse bias, when the input optical power is $55 \mu \mathrm{W}$. (d) Measured responsivity (red circle) at resonance and dark current (blue square) as the reverse bias increases.
These values are comparable to those reported for the best germanium photodiodes. ${ }^{16}$ The effect of reverse bias on resonance wavelength is particularly interesting. We first note a blueshift in the resonance wavelength when the reverse bias is increased from $0 \mathrm{~V}$ to $-2 \mathrm{~V}$ followed by a steady redshift for higher reverse voltage. For $0 \mathrm{~V}$ reverse bias, due to the lack of an external electric field, most of the photo-generated carriers recombine inside the intrinsic region and contribute to the rise in temperature of the cold cavity which causes a redshift of the resonance wavelength. As the reverse bias increases from $0 \mathrm{~V}$ to $-2 \mathrm{~V}$, more of the photo-generated carriers are swept away from the intrinsic region before they can recombine and the cavity temperature drops causing a relative blue shift in resonance wavelength. Further increases in reverse bias increase the photocurrent but cause Ohmic heating due to the movement of photo-generated carriers, redshifting the resonance wavelength once more. Using the same calculation used in the previous paragraph, we can estimate that a change in reverse bias of $-8 \mathrm{~V}$ increases the cavity temperature by $0.6 \mathrm{~K}$.

Multiplexing and demultiplexing of multiple wavelength channels typically require use of complex arrayed waveguide structures (AWGs), Echelle gratings, or passive ring resonators. In the previous work, ${ }^{10}$ we have demonstrated that the vertically coupled waveguide-cavity architecture enables the multiplexing of multiple channels without the need for additional wavelength selective components. Here, we use the same principle to achieve wavelength demutiplexing. Due to the wavelength selective nature of our detector, only one specific wavelength channel will be detected. As a result, multiple detectors can be cascaded in series and coupled to the same waveguide. To demonstrate this functionality, we have cascaded two detectors to address two different wavelength channels, as shown in Fig. 4(a). To achieve different resonance wavelengths for the detectors, the cavities were finetuned lithographically, similar to Ref. 10. Figure 4(b) shows the normalized optical transmission spectrum (in blue) of the system, with each dip in transmission corresponding to a
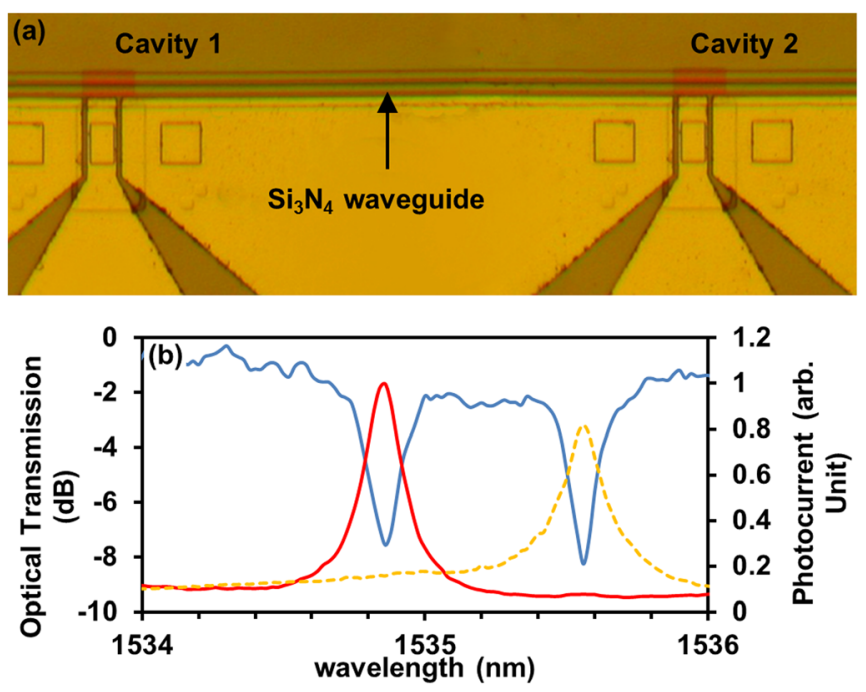

FIG. 4. (a) Top view of the cascaded PhC cavity based detector set, where two cavities are coupled to a single silicon nitride waveguide. (b) Normalized transmission spectrum (blue) of the system and measured photocurrent from detector 1 (red) and detector 2 (yellow dashed). 
cavity. Red and yellow (dashed) curves show the detected photocurrent response from the first and the second detectors, respectively. The crosstalk for channel 1 from channel 2 is $-7.2 \mathrm{~dB}$ and for channel 2 from channel 1 is $-8.5 \mathrm{~dB}$.

This approach shares many similarities with the previous work on defect-mediated detection in ring resonators. ${ }^{6,17,18}$ While the Q-factor is similar in both cases, the finesse of the $\mathrm{PhC}$ cavity is significantly higher. This provides a larger Free Spectral Range (i.e., the number of supported channels) for the same Q-factor ${ }^{10}$ and allows a smaller device reducing the capacitance, by over an order of magnitude, and thereby the power consumption of subsequent electronic amplifiers. Similar to Ref. 10, by avoiding the use of spot size converters, grating couplers, AWGs, or Echelle gratings, a significant reduction in the optical power budget is also possible, which is very promising for on-chip networks. ${ }^{19}$ This brings the effective responsivity (photocurrent relative to power from the optical fibre) of our photodiode much closer to the III-V/Germanium demultiplexed photodetection systems. Alternatively to operating as a photo-detector, the system could be considered for monitoring light propagating in a dielectric waveguide, avoiding the need for tap-off and spot size converting structures. Further optimisation of the implantation dose and coupling conditions will lead to improvements in the responsivity. Improvements in the pin junction layout and the use of sidewall passivation will also improve the extraction of photocarriers. The nonlinear dependence of responsivity in Fig. 3(b) indicates that further improvements in coupled power can lead to significant enhancements of the responsivity. This is related to the device of Ref. 13, though we use lower and more practical Q-factors.

In summary, we have demonstrated wavelength selective photo-detection in deep-level mediated silicon photodiodes. Due to the high optical confinement achievable in $\mathrm{PhC}$ cavities, the detector size can be reduced considerably in comparison to silicon waveguide based devices. Additionally, vertically coupling to low refractive index waveguides provides very low insertion losses and access to powerful passive optical components. By cascading multiple cavities along the same waveguide, a simple and elegant photo-detection scheme may be realised, which is the ideal counterpart to the modulator architecture of Ref. 10.

This work was supported by EPSRC under the UK silicon photonics project and through the EraNET Nano-Sci project LECSIN. The authors would like to thank Andrea Melloni and Emel Tasyurek for useful discussions.

${ }^{1}$ D. A. B. Miller, Proc. IEEE 97, 1166 (2009).

${ }^{2}$ H. Park, A. W. Fang, R. Jones, O. Cohen, O. Raday, M. N. Sysak, M. J. Paniccia, and J. E. Bowers, Opt. Express 15, 6044 (2007).

${ }^{3}$ L. Vivien, J. Osmond, J.-M. Fédéli, D. Marris-Morini, P. Crozat, J.-F. Damlencourt, E. Cassan, Y. Lecunff, and S. Laval, Opt. Express 17, 6252 (2009).

${ }^{4}$ A. P. Knights, J. D. B. Bradley, S. H. Gou, and P. E. Jessop, J. Vac. Sci. Technol. A 24, 783 (2006).

${ }^{5}$ M. W. Geis, S. J. Spector, M. E. Grein, R. T. Schulein, J. U. Yoon, D. M. Lennon, S. Deneault, F. Gan, F. X. Kaertner, and T. M. Lyszczarz, IEEE Photon. Technol. Lett. 19, 152 (2007).

${ }^{6}$ J. K. Doylend, P. E. Jessop, and A. P. Knights, Opt. Express 18, 14671 (2010).

${ }^{7}$ K. Debnath, K. Welna, M. Ferrera, K. Deasy, D. G. Lidzey, and L. O'Faolain, Opt. Lett. 38, 154 (2013).

${ }^{8}$ Y. Xu, Y. Li, R. K. Lee, and A. Yariv, Phys. Rev. E 62, 7389 (2000).

${ }^{9}$ K. Welna, S. L. Portalupi, M. Galli, L. O'Faolain, and T. F. Krauss, IEEE J. Quantum Electron. 48, 1177 (2012).

${ }^{10}$ K. Debnath, L. O'Faolain, F. Y. Gardes, A. G. Steffan, G. T. Reed, and T. F. Krauss, Opt. Express 20, 27420 (2012).

${ }^{11}$ P. J. Foster, J. K. Doylend, P. Mascher, A. P. Knights, and P. G. Coleman. J. Appl. Phys. 99, 073101 (2006).

${ }^{12}$ S. A. Clark, B. Culshaw, E. J. C. Dawnay, and I. E. Day, Proc. SPIE 3936, 16-24 (2000).

${ }^{13}$ T. Tanabe, H. Sumikura, H. Taniyama, A. Shinya, and M. Notomi, Appl. Phys. Lett. 96, 101103 (2010).

${ }^{14}$ R. Hayakawa, N. Ishikura, H. C. Nguyen, and T. Baba, Appl. Phys. Lett. 102, 031114 (2013).

${ }^{15}$ B. Vest, E. Lucas, J. Jaeck, R. Haidar, and E. Rosencher, Appl. Phys. Lett. 102, 031105 (2013).

${ }^{16}$ C. T. DeRose, D. C. Trotter, W. A. Zortman, A. L. Starbuck, M. Fisher, M. R. Watts, and P. S. Davids, Opt. Express 19, 24897-24904 (2011).

${ }^{17}$ D. F. Logan, P. Velha, M. Sorel, R. M. De La Rue, A. P. Knights, and P. E. Jessop, IEEE Photon. Technol. Lett. 22, 1530 (2010).

${ }^{18}$ K. Preston, Y. H. D. Lee, M. Zhang, and M. Lipson, Opt. Lett. 36, 52 (2011).

${ }^{19}$ A. Biberman, K. Preston, G. Hendry, N. Sherwood-Droz, J. Chan, J. S. Levy, M. Lipson, and K. Bergman, ACM J. Emerg. Technol. Comput. Syst. 7, 7 (2011). 